\title{
Jahn-Teller effect and electron correlation in alkali doped fullerides
}

\author{
L. F. Chibotaru* \\ Department of Chemistry, University of Leuven, Celestijnenlaan 200F, B-3001 Leuven, Belgium
}

Received 28 September 2004, accepted 15 October 2004

Published online 20 January 2005

PACS 71.10-Fd, 71.20.Tx, 71.45.Gm, 75.10.Dg

The interplay between Jahn-Teller effect and electron correlation in alkali doped fullerides is reviewed for compounds from both sides of Mott-Hubbard transition. In Mott-Hubbard fullerides the nature of low-lying excitations strongly depends on the symmetry of the compounds, corresponding either to spin (Heisenberg) type for low symmetry compounds $\left(\mathrm{K}_{3}\left(\mathrm{NH}_{3}\right) \mathrm{C}_{60}\right)$ or to spin-vibronic superexchange for cubic compounds $\left(\mathrm{Li}_{3}\left(\mathrm{NH}_{3}\right)_{6} \mathrm{C}_{60}\right)$. In the band fullerides $\left(\mathrm{A}_{3} \mathrm{C}_{60}, \mathrm{~A}_{4} \mathrm{C}_{60}, \mathrm{~A}=\mathrm{K}, \mathrm{Rb}\right)$ the Jahn-Teller effect leads to a splitting of the cubic band structure in three or two unequally populated subbands (orbital disproportionation), which gives rise to a number of features in the spectroscopy of these compounds.

() 2005 WILEY-VCH Verlag GmbH \& Co. KGaA, Weinheim

\section{Introduction}

Alkali doped fullerides have been the object of intensive investigations in the last decade [1,2]. In these crystals the electrons from alkali atoms are transferred into threefold degenerate orbitals of the fullerene sites. The relatively strong intrasite electron repulsion together with orbital degeneracy results in a concomitent presence of charge, spin, orbital and lattice (Jahn-Teller) degrees of freedom in the low-energy states. In this respect alkali doped fullerides are similar to other strongly correlated materials with orbital degeneracy [3, 4] and show a large variety of electronic phases as well. A remarkable feature of fullerides is that the band energy, the intrasite Jahn-Teller effect and the frequency of Jahn-Teller modes are within the same energy scale. As a result the Jahn-Teller effect in fullerides is manifested quite differently from other strongly correlated materials.

In the present paper we address the role played by the Jahn-Teller effect in alkali doped fullerides in two limits, the Mott-Hubbard insulator and the band metal. Typical Mott-Hubbard fullerides are $\mathrm{K}_{3}\left(\mathrm{NH}_{3}\right) \mathrm{C}_{60}[5,6]$ and $\mathrm{Li}_{3}\left(\mathrm{NH}_{3}\right)_{6} \mathrm{C}_{60}$ [7] where the amonia spacers increase the interfullerene distances and drive these crystals into a Mott-Hubbard regime. We show that different lattice symmetry, which is orthorombic for potassium and cubic for lithium compound, leads to different manifestation of the Jahn-Teller effect and as a result to different nature of magnetic interactions in these compounds. As an example of band fulleride we consider the $\mathrm{K}_{3} \mathrm{C}_{60}$ crystal, where the Jahn-Teller instability of the band structure is investigated and its manifestation in optical and EELS spectroscopies is emphasized.

\footnotetext{
Corresponding author: e-mail: Liviu.Chibotaru@chem.kuleuven.ac.be
} 


\section{The model for the LUMO band}

The starting point is the tight-binding model confined to the three degenerate $t_{1 u}$ lowest unoccupied (in undoped compounds) molecular orbitals (LUMO) on each fullerene site. The Hamiltonian contains transfer, bielectronic and vibronic parts [8]:

$$
\begin{aligned}
\mathcal{H}=\mathcal{H}_{t}+\mathcal{H}_{\mathrm{bi}}+\mathcal{H}_{\mathrm{vib}}, \quad \mathcal{H}_{t}=\sum_{\boldsymbol{m}, \boldsymbol{n}} \sum_{i, j, \sigma} h_{\boldsymbol{m} i \boldsymbol{n} j} a_{\boldsymbol{m}, i \sigma}^{\dagger} a_{\boldsymbol{n}, j \sigma}, \\
\mathcal{H}_{b i}=\frac{1}{2} \sum_{\boldsymbol{m}} \sum_{i, \sigma}\left[U_{\|} n_{\boldsymbol{m}, i \sigma} n_{\boldsymbol{m}, i-\sigma}+U_{\perp} \sum_{j \neq i} \sum_{\sigma^{\prime}} n_{\boldsymbol{m}, i \sigma} n_{\boldsymbol{m}, j \sigma^{\prime}}\right. \\
\left.-J \sum_{j \neq i}\left(n_{\boldsymbol{m}, i \sigma} n_{\boldsymbol{m}, j \sigma}-a_{\boldsymbol{m}, i \sigma}^{\dagger} a_{\boldsymbol{m}, j \sigma} a_{\boldsymbol{m}, j-\sigma}^{\dagger} a_{\boldsymbol{m}, i-\sigma}-a_{\boldsymbol{m}, i \sigma}^{\dagger} a_{\boldsymbol{m}, j \sigma} a_{\boldsymbol{m}, j-\sigma}^{\dagger} a_{\boldsymbol{m}, i-\sigma}\right)\right], \\
\mathcal{H}_{\mathrm{vib}}=\sum_{\boldsymbol{m}} \sum_{\mu, \gamma}\left[\frac{1}{2} K_{\mu H} q_{\boldsymbol{m} \mu \gamma}^{2}+\sqrt{\frac{3}{5}} V_{\mu H} \sum_{i, j, \sigma} G_{i j}^{\gamma} a_{\boldsymbol{m}, i \sigma}^{\dagger} a_{\boldsymbol{m}, j \sigma} q_{\boldsymbol{m} \mu \gamma}\right],
\end{aligned}
$$

Here, $i, j=x, y, z$ are degenerate components of the active $t_{1 u}$ orbital triplet at each site $\boldsymbol{m}$, and $\sigma=\alpha, \beta$ denotes the spin components. $\mathcal{H}_{t}$ accounts for the transfer type interactions between nearest neighbours. The hopping amplitudes are obtained via the fitting of the LDA band structure [9]. $\mathcal{H}_{\mathrm{bi}}$ is limited to intramolecular bielectronic interactions. An atomic-like relation $U_{\|}-U_{\perp}=2 J$ can be shown to hold. For pristine fullerene crystals $U_{\|} \approx 0.8 \div 1.5 \mathrm{eV}$ and $J \approx 0.03 \div 0.05 \mathrm{eV}$ [1]. In doped crystals $U_{\|}$ is further reduced by the effects of screening due to the LUMO band. Finally, the vibronic part consists of the interaction with the effective on-site quadrupolar type nuclear distortions $\left\{q_{m \mu \gamma}\right\}$, where $\mu$ runs over the eight $H_{g}$ modes and $\gamma=\theta, \varepsilon, \xi, \eta, \zeta$ denotes the components. The $G_{i j}^{\gamma}$ quantities are the Clebsch-Gordan coefficients for the $T_{1} \otimes T_{1}=H$ product. The frequencies of the eight vibrational modes are within the range $270 \div 1600 \mathrm{~cm}^{-1}$. The energy of the Jahn-Teller stabilization for $\mathrm{C}_{60}^{-}$, $E_{H}=1 / 5 \sum_{\mu} V_{\mu H}^{2} / K_{\mu H}$, was found to be $0.1 \mathrm{eV}$ in photoemission [1]. For $\mathrm{C}_{60}^{3-}$ we have $E_{\mathrm{JT}}^{3}=3 E_{H}$ [10] and one should expect $E_{\mathrm{JT}}^{3}=0.3 \mathrm{eV}$. Actually this value is twice smaller which points to a significant reduction of vibronic constants upon doping. For an average frequency of $H_{g}$ modes we have the ratio $\bar{\omega}_{H} / E_{H} \sim 1$, i.e. the fullerenes are in the intermediate coupling regime [11].

\section{Mott-Hubbard fullerides}

In these compounds the ratio between the LUMO bandwidth $W$ and the Hubbard $U$ is small and the ground state corresponds to a Mott-Hubbard insulator for stoichiometric doping. The LUMO electrons are mostly localized at fullerene sites and the gain of kinetic energy is achieved by their partial delocalization over neighbour sites. This gain has precisely the same origin as the antiferromagnetic superexchange mechansim, $J_{a f}=4 t^{2} / U$, where $t$ is the intersite transfer parameter, and is quite small in MottHubbard fullerides. Indeed, for the b.c.c. $\mathrm{Li}_{3}\left(\mathrm{NH}_{3}\right)_{6} \mathrm{C}_{60}$ we have [7]: $W=0.3 \mathrm{eV}, t \approx W /(2 \times 9)=0.015 \mathrm{eV}$, $U \approx 1 \mathrm{eV}$, and therefore $J_{a f} \approx 0.001 \mathrm{eV}$.

Within each fullerene site $\mathrm{C}_{60}^{3-}$ there is a competition between Hund's rule coupling, which tends to align the three electrons ferromagnetically (Fig. 1a), and the Jahn-Teller effect which tends to populate consecutively the splitted orbitals (Fig. 1b). To compare the energies of these two electronic configurations we should take also into account the change of vibrational energy in the Jahn-Teller configuration [11]. Treating the Jahn-Teller effect as a one-mode problem $\left(\bar{\omega}_{\mathrm{H}}\right)$ in the strong coupling limit [11] we obtain $3 U_{\|}-9 J+5 / 2 \hbar \bar{\omega}_{\mathrm{H}}$ for the Hund's configuration and $3 U_{\|}-4 J-E_{\mathrm{JT}}^{3}+\hbar \bar{\omega}_{\mathrm{H}}$ for the Jahn-Teller configuration. The gain of $3 / 2 \hbar \bar{\omega}_{\mathrm{H}}$ in the latter case is due to the fact that the Jahn-Teller adiabatic potential for $\mathrm{C}_{60}^{3-}$ has a threedimensional continuum of minima (a trough) in the ground state [10, 12]. With the estimation of the parameters given above we conclude that the Jahn-Teller configuration should be 
a)

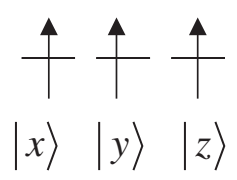

b)

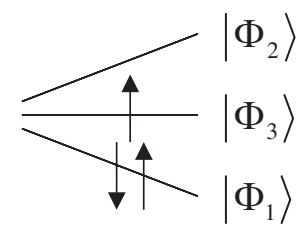

Fig. 1 Hund's (a) and Jahn-Teller (b) electronic configurations of $\mathrm{C}_{60}^{3-} \cdot\left|\Phi_{i}\right\rangle$ are adiabatic orbitals.

the lowest in energy. It was found indeed that the ground state spin of fullerenes in $\mathrm{Li}_{3}\left(\mathrm{NH}_{3}\right)_{6} \mathrm{C}_{60}$ [7] and $\mathrm{K}_{3}\left(\mathrm{NH}_{3}\right) \mathrm{C}_{60}[5,6]$ corresponds to $S=1 / 2$.

The threedimensional trough on the lowest energy surface of $\mathrm{C}_{60}^{3-}$ leads to a complex nuclear dynamics, involving free rotations of Jahn-Teller deformations along three angular coordinates and harmonic vibrations along another degrees of freedom. The solutions for low-lying states in the strong coupling approximation are $[10,12]$ :

$$
\begin{aligned}
& \Psi(r, q, \alpha, \gamma, \vartheta, \varphi)=\Psi^{a d}(r, \gamma, \vartheta, \varphi) \Psi_{n_{1}, n_{2}}^{\mathrm{vib}}(q, \alpha) \Psi_{L, M, K}^{\mathrm{rot}}(\gamma, \vartheta, \varphi), \\
& E_{n_{1} n_{2} L K}=-E_{\mathrm{JT}}^{3}+\hbar \bar{\omega}_{\mathrm{H}}\left(n_{1}+n_{2}+1\right)+\frac{\hbar^{2}}{8 q_{0}^{2}}+\frac{\hbar^{2}}{2 q_{0}^{2}}\left[L(L+1)-\frac{3}{4} K^{2}\right],
\end{aligned}
$$

where $r$ denotes electronic coordinates, $q$ is the amplitude of the $H_{g}$ vibrations, $q_{0}$ is the equilibrium value in the trough, and $\gamma, \vartheta, \varphi$ are three Euler angles specifying the rotation in the trough. $\Psi^{a d}$ is the adiabatic electronic function, describing the electronic state at fixed nuclear coordinates; $\Psi^{\text {vib }}$ describes twodimensional vibrations across the trough; $\Psi_{L, M, K}^{\text {rot }}$ is the rotational part, coinciding with the solution for a symmetric top with $K$ even. The ground state corresponds to $L=1, K=0$,

$$
\Psi_{1, M, 0}^{\mathrm{rot}}=\sqrt{\frac{2}{\pi}} Y_{1 M}(\vartheta, \varphi),
$$

and is therefore threefold degenerate. The nature of the ground state does not change when going beyond strong coupling approximation and when multiplet effects are taken into account [10,12].

The lowest vibronic states in Eq. (2) correspond to excitations in the rotational sector at $n_{1}=n_{2}=0$. With $q_{0}=\sqrt{3} V_{\mathrm{H}} / K_{\mathrm{H}}$, the rotational quantum can be estimated as $\Delta E_{\mathrm{rot}}=\frac{1}{4}\left(\hbar \bar{\omega}_{\mathrm{H}}\right)^{2} / E_{\mathrm{JT}}^{3} \sim \frac{1}{4} \hbar \bar{\omega}_{\mathrm{H}}$. In cubic fullerides like $\mathrm{Li}_{3}\left(\mathrm{NH}_{3}\right)_{6} \mathrm{C}_{60}$ the fullerene sites are in high symmetry positions and the electronic degeneracy as well as the vibronic degeneracy is not removed. Since $J_{a f}$ was found to be much smaller than the intervals in the rotational vibronic spectrum, we may conclude that in cubic fullerides the superexchange interaction takes place between degenerate ground vibronic states on fullerene sites [13]. On the other hand, if an additional low symmetry crystal field is present as in orthorombic $\mathrm{K}_{3}\left(\mathrm{NH}_{3}\right) \mathrm{C}_{60}$ the system can be localized in the trough arround some fixed values of $\gamma, \vartheta, \varphi$. This localization occurs when low symmetry crystal field components exceed the rotational quantum. In such a case the superexchange takes place between nondegenerate electronic configurations (Fig. 1b) corresponding to fixed values of Euler angles.

The superexchange Hamiltonian is obtained in the following general form:

$$
\mathcal{H}_{\text {eff }}=\mathcal{H}_{0}+\mathcal{H}^{\prime}+\frac{1}{U} \mathcal{H}_{t} \cdot \mathcal{H}_{t}-\frac{1}{2 U^{2}}\left[\left[\mathcal{H}^{\prime}, \mathcal{H}_{t}\right], \mathcal{H}_{t}\right],
$$

where $\mathcal{H}_{0}$ contains all intrafullerene interactions depending on the total occupation number, with an effective $U$, and $\mathcal{H}^{\prime}$ includes the rest of intrasite interactions. The Hamiltonian (4) is expressed via spin and orbital operators in a standard way [14]. This procedure is general and permits, in particular, to fully take into account the Jahn-Teller splitting.

In the case of low symmetry Mott-Hubbard fullerides we have to average $\mathcal{H}_{\text {eff }}$ over the nondegenerate electronic configurations with fixed adiabatic orbitals on fullerene sites (Fig. 1b). The resulting op- 
erator describes the Heisenberg exchange interaction:

$$
\begin{aligned}
H_{\mathrm{ex}}= & -\frac{t_{12}^{2}+t_{21}^{2}}{U}\left(2-\frac{4 E_{\mathrm{JT}}^{3}+2 J}{3 U}\right)-\frac{t_{13}^{2}+t_{31}^{2}}{U}\left(1+\frac{2 E_{\mathrm{JT}}^{3}-5 J}{6 U}\right) \\
& -\frac{t_{23}^{2}+t_{32}^{2}}{U}\left(1+\frac{2 E_{\mathrm{JT}}^{3}-17 J}{6 U}\right)-\frac{t_{33}^{2}}{U}\left(1+\frac{4 E_{\mathrm{JT}}^{3}-10 J}{3 U}\right) \\
& +\left[4 \frac{t_{33}^{2}}{U}\left(1+\frac{4 E_{\mathrm{JT}}^{3}-10 J}{3 U}\right)-2 \frac{t_{13}^{2}+t_{31}^{2}+t_{23}^{2}+t_{32}^{2}}{U} \frac{J}{U}\right] S_{1} \cdot S_{2} .
\end{aligned}
$$

In this equation, each transfer parameter $t_{i j}(i$ and $j$ denote the adiabatic orbitals) depends on six Euler angles. The antiferromagnetic contribution is determined by the transfer parameter between half filled orbitals, $t_{33}$. Therefore the exchange is ferromagnetic for $t_{33}=0$. This seems to be the case in $\mathrm{K}_{3}\left(\mathrm{NH}_{3}\right) \mathrm{C}_{60}$. Indeed, the low temperature measurements show that the spins arrange in this compound antiferromagnetically along the translational directions (similar orbitals on neighbour sites overlap) and ferromagnetically in perpendicular directions [6].

In the case of cubic fullerides we have to average $\mathcal{H}_{\text {eff }}$ over degenerate vibronic states (3) of fullerene sites. Therefore the superexchange operator of a given pair is a $9 \times 9$ matrix in the space of vibronic states of the two sites, with spin operators of Heisenberg type as matrix elements. We face here the phenomenon of spin-vibronic exchange interaction [13] which resembles the spin-orbital superexchange [15] but involves vibronic rather than orbital states of the sites. To be specific we consider the b.c.c. compound $\mathrm{Li}_{3}\left(\mathrm{NH}_{3}\right)_{6} \mathrm{C}_{60}$ [7]. In this crystal each pair of fullerene sites is found on a threefold rotational axis. Choosing the quantization axis along the axis of the pair, the electron transfer within the pair will conserve the orbital quantum number of the sites. Therefore $\mathcal{H}_{t}$ contains only two transfer parameters, of $\sigma\left(t_{0}\right)$ and $\pi\left(t_{1}\right)$ type. Similarly, the spin-vibronic superexchange interaction will conserve the projection of the total vibronic momentum on the axis of the pair $I=M_{1}+M_{2}$. As in the case of spin-orbital interaction, the obtained Hamiltonian can be expressed via pseudospin and quadrupole operators [16], which act now in the space of vibronic states of the sites. Therefore the spin-vibronic superexchange for $\mathrm{C}_{60}^{3-}-\mathrm{C}_{60}^{3-}$ formally resembles the spin-orbital interaction for a $t_{2 g}^{1}-t_{2 g}^{1}$ pair. The only difference is in the constants in front of similar terms of the two Hamiltonians.

Figure 2 compares the spin-vibronic and spin-orbital pair spectra for a threefold degeneracy of sites (the Jahn-Teller effect is absent $n$ the second case). One can see two main differences. First, the splitting
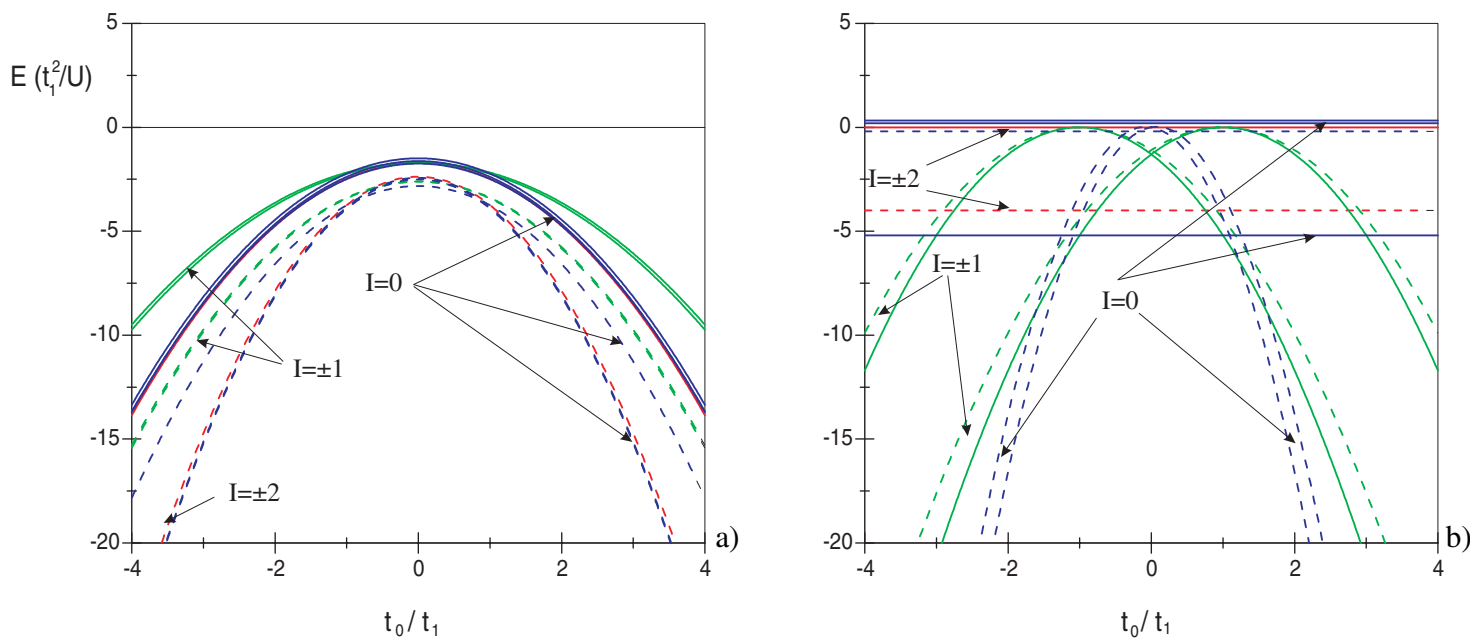

Fig. 2 (online colour at: www.pss-b.com) Spin-vibronic spectrum for $\mathrm{C}_{60}^{3-}-\mathrm{C}_{60}^{3-}$ at $J / U=0.05, E_{\mathrm{JT}}^{3} / U=0.15$ (a) and spin-orbital spectrum for a $t_{2 g}^{1}-t_{2 g}^{1}$ pair at $J / U=0.1$ (b) in a b.c.c. lattice. Solid lines correspond to $S=1$ and dashed lines to $S=0$ ( $S$ is the spin of the pair). 
of the spin levels in the spin-vibronic case is $3 \div 4$ times smaller than in the spin-orbital case. We encounter here a vibronic reduction of superexchange interactions [13], which is nothing but Ham reduction factor for electronic observables in Jahn-Teller systems [11]. Second, while the ground state of the spin-orbital pair can be both ferro and antiferromagnetic, the ground state of the spin-vibronic pair is always antiferromagnetic. This is supported by the antiferromagnetic susceptibility observed in $\mathrm{Li}_{3}\left(\mathrm{NH}_{3}\right)_{6} \mathrm{C}_{60}$ [7].

\section{Band fullerides}

Now we treat the LUMO Hamiltonian (1) in the band limit. Supposing arbitrary static Jahn-Teller distortions of each $\mathrm{C}_{60}$, their amplitudes can be expressed exactly through the elements of the intrasite oneparticle density matrix, $D_{m i \sigma}^{m j \sigma}$ ( $\boldsymbol{m}$ denotes sites and $i, j$ are $t_{1 u}$ orbital components) [8]. The resulting Fock operator, describing band orbitals with symmetry breaking in the orbital sector, has the following form in the site representation:

$$
\begin{aligned}
& F_{m i n n j}=h_{m i ; n j}+\frac{5}{6}\left(U_{\|}-2 J\right) n \delta_{i j} \delta_{m, n}-U_{d} \delta_{m, n}\left[\Delta N_{m i \sigma} \delta_{i j}+D_{m i \sigma}^{n j \sigma}\left(1-\delta_{i j}\right)\right], \\
& U_{d}=U_{\|}+6 E_{H}-5 J
\end{aligned}
$$

where $n$ is the average population on the $t_{1 u}$ shell $\left(n=3\right.$ for $\left.\mathrm{K}_{3} \mathrm{C}_{60}\right)$ and $\Delta N_{m i \sigma}$ is the deviation of the population of the orbital component $i$ (with spin $\sigma=\alpha$ or $\beta$ ) from the cubic symmetry value (1/2). The merohedral disorder was simulated by a random distribution of two standard orientations of fullerene molecules in an enlarged unit cell containing 256 sites [17]. The next-LUMO $\left(t_{1 g}\right)$ band was described by the tight binding Hamiltonian giving the dispersion and the shift relative to the $t_{1 u}$ band in agrement with conventional LDA calculations [9].

The resulting density of states (DOS) for $\mathrm{K}_{3} \mathrm{C}_{60}$ is shown in Fig. 3 [17]. Starting from the cubic electron distribution $\left(\Delta N_{m i \sigma}=0\right)$ we obtain for the $t_{1 u}$ band a structureless DOS profile (left pannel) coinciding with previous results [18]. Nonequal population of the $t_{1 u}$ orbital components in the initial electron distribution $\left(\Delta N_{m i \sigma} \neq 0\right)$ results in the splitting of the cubic band into three subbands (right pannel). Each
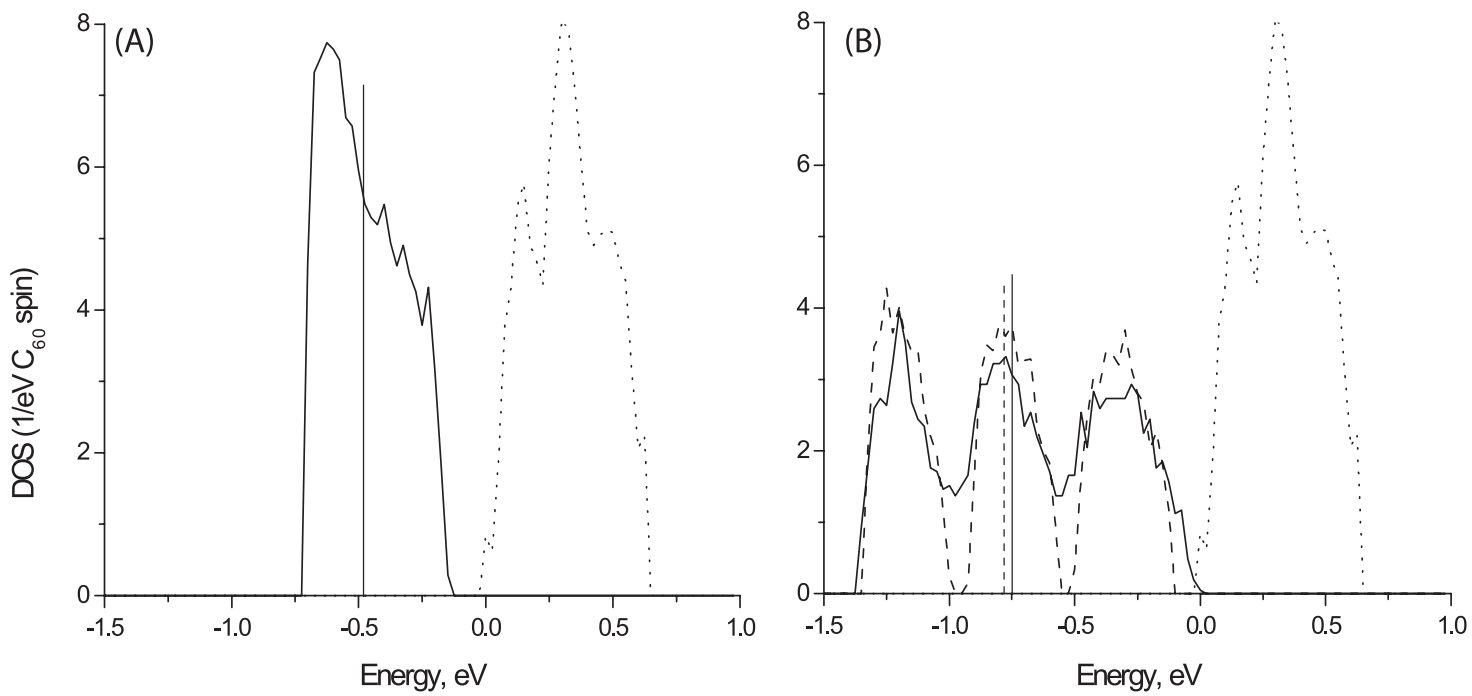

Fig. 3 Density of states of the the LUMO and next-LUMO bands in $\mathrm{K}_{3} \mathrm{C}_{60}$ obtained by conventional (left) and broken-symmetry (right) band structure calculations including merohedral disorder. In the right pannel the $t_{1 u}$ band is resolved into three subbands: the fully occupied (lowest), the half filled (middle) and the empty (upper) shown for the case of translational order (solid lines) and full disorder (dashed lines) of the corresponding orbital components on the sites. The $t_{1 g}$ band is shown by dotted line. Vertical lines correspond to the Fermi level. 

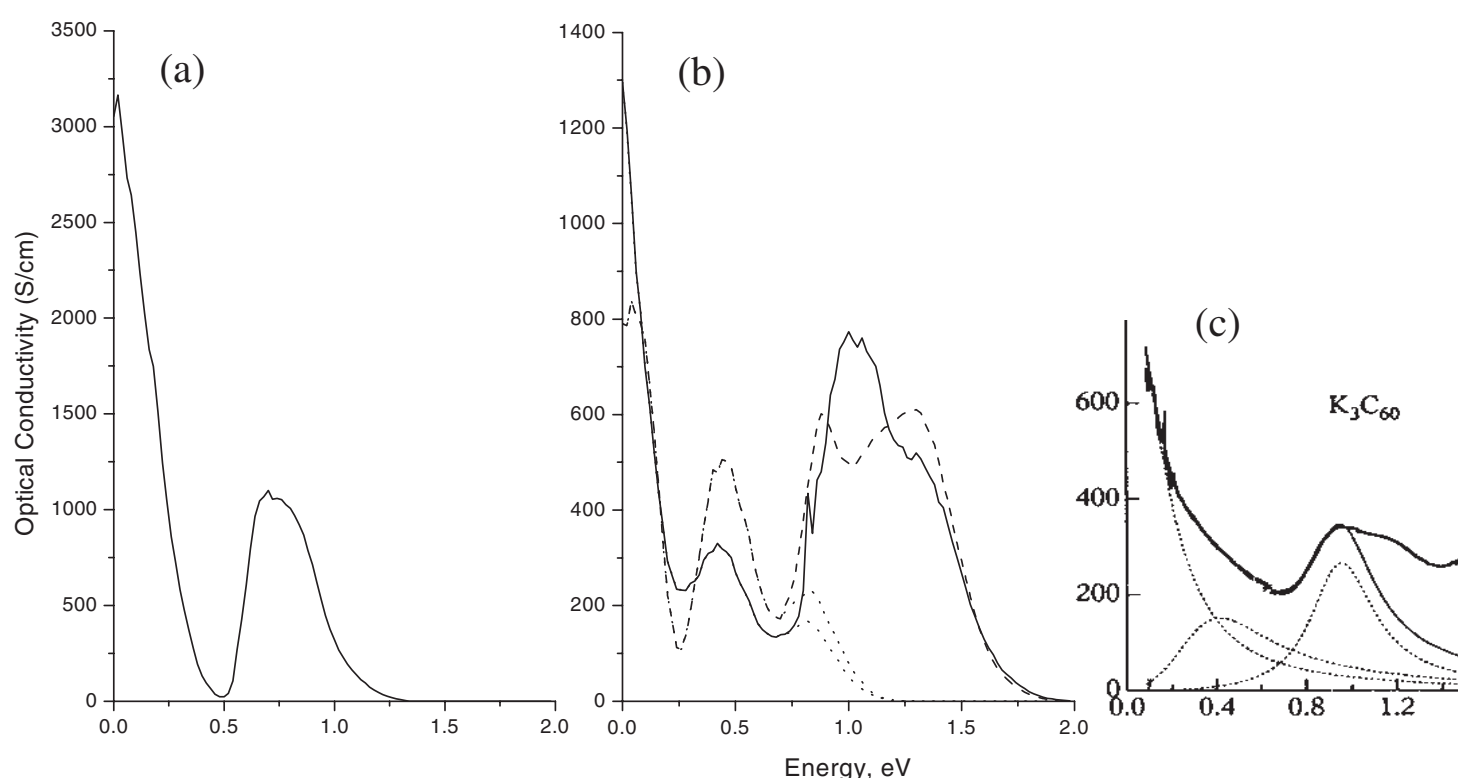

Fig. 4 Conductivity of $\mathrm{K}_{3} \mathrm{C}_{60}$ : conventional band structure calculations (left), broken-symmetry calculations (middle) and experiment [21] (right). Solid and dashed lines in the middle pannel correspond to translational order and full disorder of disproportionated $t_{1 u}$ orbitals at fullerene sites. Dotted lines show the intra $t_{1 u}$ band contribution to the conductivity in the overlapping region.

$t_{1 u}$ orbital component on fullerene sites contributes almost exclusively to one of the three subbands. Therefore the relative shift of the subbands is described by the difference of the corresponding terms $U_{d} \Delta N_{m i \sigma}$ in Eq. (6). The magnitude of these shifts is much exaggerated at the Hartree-Fock level of calculations but becomes less pronounced when the screening of the exchange interaction between band states is taken into account, as it was shown by $G W$ calculations for $\mathrm{K}_{4} \mathrm{C}_{60}$ [19]. Accordingly, in the present calculations we consider the shift of the side subbands relative to the middle one as a free parameter (the only one) used to fit the position of the lowest subband in Fig. $3 \mathrm{~b}$ to the broad peak in the photoemission spectrum [20].

Figure 4 shows the calculated optical conductivity spectra together with the experimental one taken from Ref. [21]. The intra $t_{1 u}$ band contribution to the optical conductivity in the conventional band structure calculation (Fig. 4a) looks like a broad Drude peak whereas in the broken-symmetry aproach (Fig. 4b) it contains two additional absorption bands corresponding to transitions between neighbour subbands $(\approx 0.4 \mathrm{eV})$ and between the lower and upper subbands $(\approx 0.8 \mathrm{eV})$. The transition from the middle $t_{1 u}$ subband to $t_{1 g}$ is almost superimposed on the highest $t_{1 u} \rightarrow t_{1 u}$ absorption band while the transition from the lowest $t_{1 u}$ subband to $t_{1 g}$ gives the third absorption band at $\approx 1.3 \mathrm{eV}$. These three bands coincide well with experiment (Fig. 4c). Moreover the $d c$ limit in Fig. 4b matches the measured value of $1300 \mathrm{~S} / \mathrm{cm}$ in Ref. [22]. The calculations based on the conventional approach (Fig. 4a) fail to reproduce these features.

The calculated optical conductivity was further used to simulate the electron loss function via the Drude-Lorentz fit of the spectra in Fig. 4. [23]. Applying such a transformation to the experimental conductivity in Fig. 4c gives the position and the width of the plasmon peak which are in good agreement with the EELS data [24]. Compared to it the peak which results from conventional band structure calculations is placed too high in energy, is several times more intensive and is much narrower. By contrast broken-symmetry calculations give a plasmon peak closer to the right position and enlarged by shoulders corresponding to transitions between $t_{1 u}$ subbands, so that the intensity and the width of the plasmon band match well the experiment [23]. 


\section{Conclusions}

We studied the manifestation of the Jahn-Teller effect in alkali doped fullerides in two limits.

In Mott-Hubbard fullerides, the gain of the rotation energy of Jahn-Teller deformations in the trough of the lowest potential energy surface of $\mathrm{C}_{60}$ ions exceeds the gain of kinetic energy of electron delocalization between neighbour fullerene sites. This is the specific of fullerene materials which is due to the fact that the $\mathrm{C}_{60}$ ions are in an intermediate vibronic coupling regime and the frequencies of active vibrations are quite high. As a result in cubic fullerides the superexchange interaction takes place between threefold degenerate vibronic ground states of $\mathrm{C}_{60}^{3-}$ sites (spin-vibronic superexchange). In lower symmetry fullerides the Jahn-Teller deformations on each fullerene site are localized and the superexchange becomes of Heisenberg type.

In band fullerides the intrasite Jahn-Teller effect and the electron correlation act together to split the cubic LUMO band in several unequally populated subbands (orbital disproportionation). This allows to explain qualitatively the photoemission, optical conductivity and EELS profiles in $\mathrm{A}_{3} \mathrm{C}_{60}$ and $\mathrm{A}_{4} \mathrm{C}_{60}$. The mean-field broken-symmetry approach is obviously oversimplified and should be completed by a proper account of other correlation and vibronic effects. However the ability to catch basic spectroscopic features allows us to conclude that the obtained solutions are a good starting point for these fullerides.

Acknowledgement This work has been supported by the Belgian Science Foundation and Flemish Government under Concerted Action Scheme.

\section{References}

[1] O. Gunnarsson, Rev. Mod. Phys. 69, 575 (1997).

[2] M. J. Rosseinsky, Chem. Mater. 10, 2665 (1998).

[3] M. Imada, A. Fujimori, and Y. Tokura, Rev. Mod. Phys. 70, 1039 (1998).

[4] Y. Tokura and N. Nagaosa, Science 288, 462 (2000).

[5] Y. Iwasa, H. Shimoda, T. T. M. Palstra, Y. Maniwa, O. Zhou, and T. Mitani, Phys. Rev. B 53, R8836 (1996).

[6] S. Margadonna and K. Prassides, J. Solid State Chem. 168, 639 (2002).

[7] P. Durand, G. R. Darling, Y. Dubitsky, A. Zaopo, and M. J. Rosseinsky, Nature Mater. 2, 605 (2003).

[8] L. F. Chibotaru and A. Ceulemans, Phys. Rev. B 53, 15522 (1996).

[9] S. C. Erwin and W. E. Pickett, Science 254, 842 (1991).

[10] A. Auerbach, N. Manini, and E. Tosatti, Phys. Rev. B 49, 12998 (1994).

[11] I. B. Bersuker and V. Z. Polinger, Vibronic Interactions in Molecules and Crystals (Springer, Berlin, 1989).

[12] M. C. M. O’Brien, Phys. Rev. B 53, 3775 (1996).

[13] L. F. Chibotaru, submitted to Phys. Rev. Lett.

[14] L. F. Chibotaru, unpublished.

[15] K. I. Kugel and D. I. Khomskii, Sov. Phys. Usp. 25, 231 (1982).

[16] G. Khaliullin and S. Okamoto, Phys. Rev. B 68, 205109 (2003).

[17] L. F. Chibotaru and A. Ceulemans, in: Electron-Phonon Dynamics and Jahn-Teller effect, edited by G. Bevilacqua et al. (World Scientific, Singapore, 1999), p. 233.

[18] I. I. Mazin et al., Phys. Rev. Lett. 70, 4142 (1993).

[19] L. F. Chibotaru, A. Ceulemans, and S. P. Cojocaru, Phys. Rev. B 59, R12728 (1999).

[20] M. Knupfer et al., Phys. Rev. B 47, 13944 (1993).

[21] Y. Iwasa and T. Kaneyasu, Phys. Rev. B 51, 3678 (1995).

[22] L. Degiorgi et al., Phys. Rev. B 49, 7012 (1994).

[23] L.F. Chibotaru and A. Ceulemans, in: Electronic Properties of Molecular Nanostructures: XIV International Winterschool/Euruconference, edited by H. Kuzmany et al., AIP Conf. Proc. No 544 (AIP, Melville, 2000), p. 19.

[24] M. Knupfer, J. Fink, and J. F. Armbruster, Z. Phys. B 101, 57 (1996). 